\title{
Vascular pectoralis minor syndrome as an overlooked condition: A case report
}

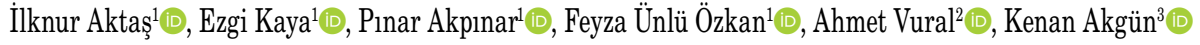 \\ ${ }^{1}$ Department of Physical Medicine and Rehabilitation, Health Sciences University, Fatih Sultan Mehmet Training and Research Hospital, Istanbul, Turkey \\ ${ }^{2}$ Department of Radiology, Health Sciences University Fatih Sultan Mehmet Training and Research Hospital, Istanbul, Turkey \\ ${ }^{3}$ Department of Physical Medicine and Rehabilitation, Istanbul University Cerrahpaşa Faculty of Medicine, Istanbul, Turkey
}

Received: January 03, 2021 Accepted: April 15, 2021 Published online: December 01, 2021

\begin{abstract}
Thoracic outlet syndrome is characterized by pain, paresthesia, muscle weakness, and arterial/venous symptoms caused by compression of the neurovascular structures. Compression mainly occurs at three distinct areas in the thoracic outlet: the retropectoralis minor space, the costoclavicular space, and the interscalene triangle. As the symptoms of these three compression sites are very similar, it is difficult to pinpoint the location of the compression and the treatment methods are quite different. Ultrasound-guided diagnostic injections play an important role in the differential diagnosis. Herein, we report a 49 -year-old female patient who was previously diagnosed with thoracic outlet syndrome and scheduled for decompression of cervical ribs, but cured by conservative methods after being diagnosed with pectoralis minor syndrome.
\end{abstract}

Keywords: Brachial plexus, pectoralis minor syndrome, thoracic outlet syndrome.

Thoracic outlet syndrome (TOS) is a neurovascular disorder caused by compression of the brachial plexus and/or the subclavian vessels. Compression may occur at three distinct areas in the thoracic outlet: retropectoralis minor space, the costoclavicular space, and the interscalene triangle. There is a wide spectrum of clinical presentations with symptoms of pain, weakness, paresthesia, numbness, coldness, tingling, and discoloration in the upper extremity. ${ }^{[1]}$ It is important to identify the location of the neurovascular compression to achieve an effective treatment and, therefore, a detailed history and physical examination are needed. Neurogenic TOS is the most common form, whereas approximately 3 to $5 \%$ are venous and $1 \%$ is arterial. ${ }^{[2]}$

Pectoralis minor syndrome (PMS) is caused by the compression of the neurovascular bundle at the retropectoralis minor space. It may stem from a congenital abnormal costa, poor posture, repetitive movement of shoulders and arms, and use of backpack. Exercises that stretch the pectoral muscles in daily living activities are also important in the etiology. It is usually seen in occupations subject to repetitive stress injury and in athletes who are engaged in sports such as rowing, swimming, shot put, weightlifting and javelin. ${ }^{[1]}$

In this article, we present a case of PMS who was previously diagnosed with TOS and scheduled for decompression of cervical ribs, but cured with conservative methods after the pectoralis minor (PM) muscle block.

\section{CASE REPORT}

A 49-year-old woman who was previously diagnosed with right TOS and scheduled for decompression of cervical ribs was referred to our clinic to attend to

Corresponding author: Pınar Akpınar, MD. SBÜ Fatih Sultan Mehmet Ĕ̆itim ve Araştırma Hastanesi, Fiziksel Tip ve Rehabilitasyon Kliniği, 34752 İçerenköy, Ataşehir, İstanbul, Türkiye. e-mail: pinar.pinarakpinar@gmail.com

Aktaş İ, Kaya E, Akpınar P, Ünlü Özkan F, Vural A, Akgün K. Vascular pectoralis minor syndrome as an overlooked condition: A case report. Turk J Phys Med Rehab 2021;67(4):538-541. 
rehabilitation practices before the operation. She was a housewife and had complaints of pain, numbness, and tingling in right upper extremity. She had no history of trauma, although her symptoms aggravated after exercise. She was particularly interested in rowing and playing tennis. She was diagnosed with cervical disc herniation two months ago. Magnetic resonance imaging (MRI) of the cervical spine revealed central disc protrusion at C4-C5, C5-C6 intervertebral levels. Right upper extremity arterial venous system color Doppler ultrasonography (USG) revealed a decrease in flow velocity and increase in the acceleration time in the axillary artery with Adson maneuver (Figure 1). Her clinical examination revealed normal and painless cervical, shoulder, and elbow range of motion in all directions with normal manual muscle testing and neurological examination.

Pain intensity was evaluated using the Visual Analog Scale (VAS) (1-10) and upper extremity function was evaluated using Quick Disabilities of the Arm, Shoulder, and Hand (QuickDASH) questionnaire. Her VAS score was 7 and the QuickDASH score was 59.1 at the time of admission. Electrophysiological examination including electromyography (EMG) was normal, ruling out cervical radiculopathy, brachial plexopathy entrapment neuropathies, and neurogenic
TOS. The Adson and Roos tests were positive at the right side. Her symptoms worsened, when pressure was applied over the right PM muscle. A diagnostic PM block was performed under the USG guidance, where $4 \mathrm{~mL} 1 \%$ lidocaine was injected into the right PM. Diagnosis of PMS was confirmed with complete resolution of her symptoms after the USG-guided PM block.

A rehabilitation program consisting of PM stretching, shoulder stabilization, postural correction exercises and shoulder proprioceptive neuromuscular facilitation was initiated five times a week, for a total of eight weeks. Hot pack (20 min), conventional transcutaneous electrical nerve stimulation $(20 \mathrm{~min})$, and therapeutic ultrasound (10 $\mathrm{min}$ ) were additionally applied to the pectoral area and shoulder girdle in each session. She was also instructed to avoid repetitive arm and shoulder movements and to take a break from rowing and tennis.

Her symptoms subsided with complete resolution at the end of the treatment. Control upper extremity arterial venous system color Doppler USG was normal (Figure 2) two months after the treatment. The patient has been symptom-free for two years. A written informed consent was obtained from the patient.

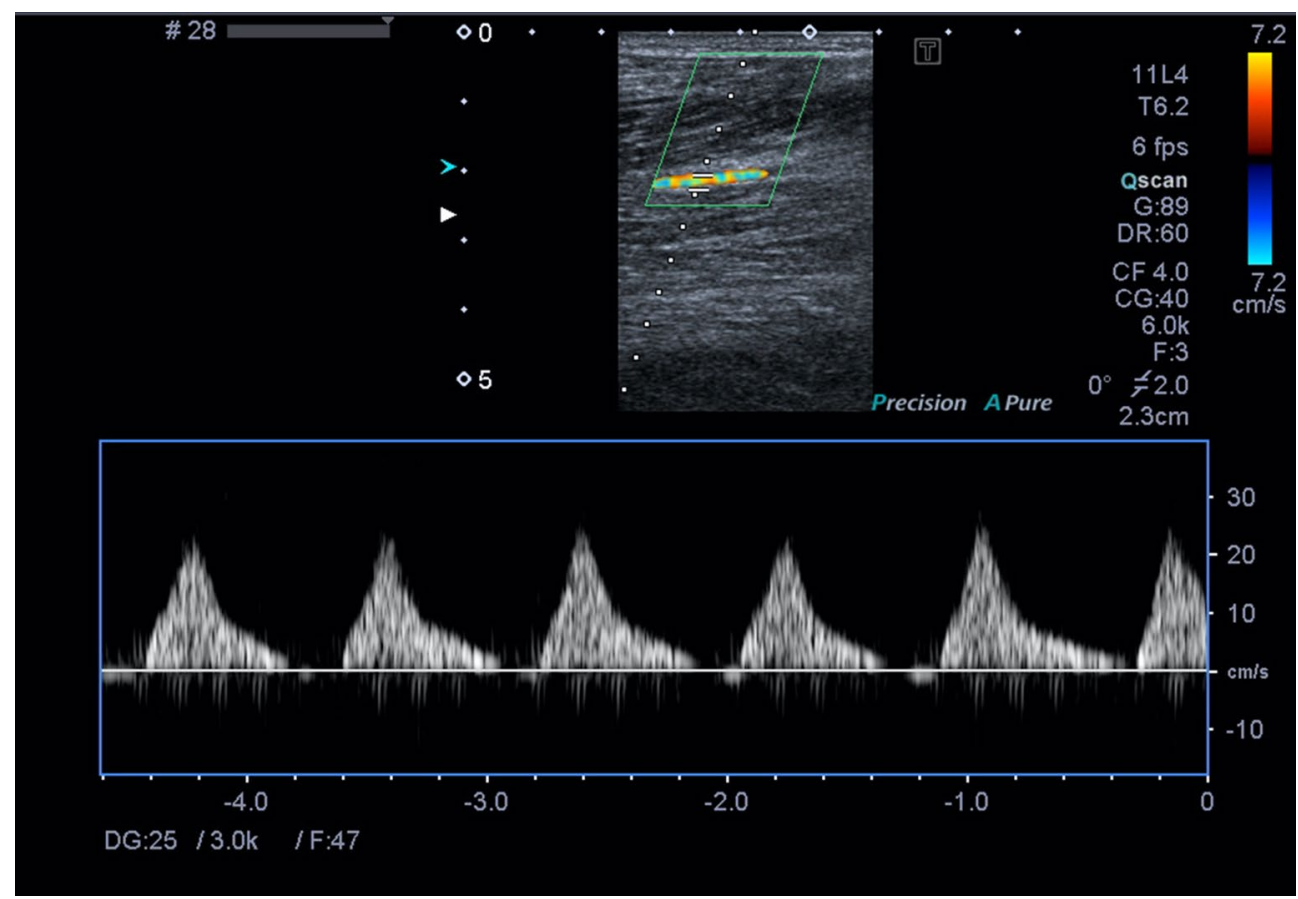

Figure 1. Color Doppler ultrasound image showing decreased flow velocity, and increased acceleration time and systolic blunting in the axillary artery on hyperabduction. 


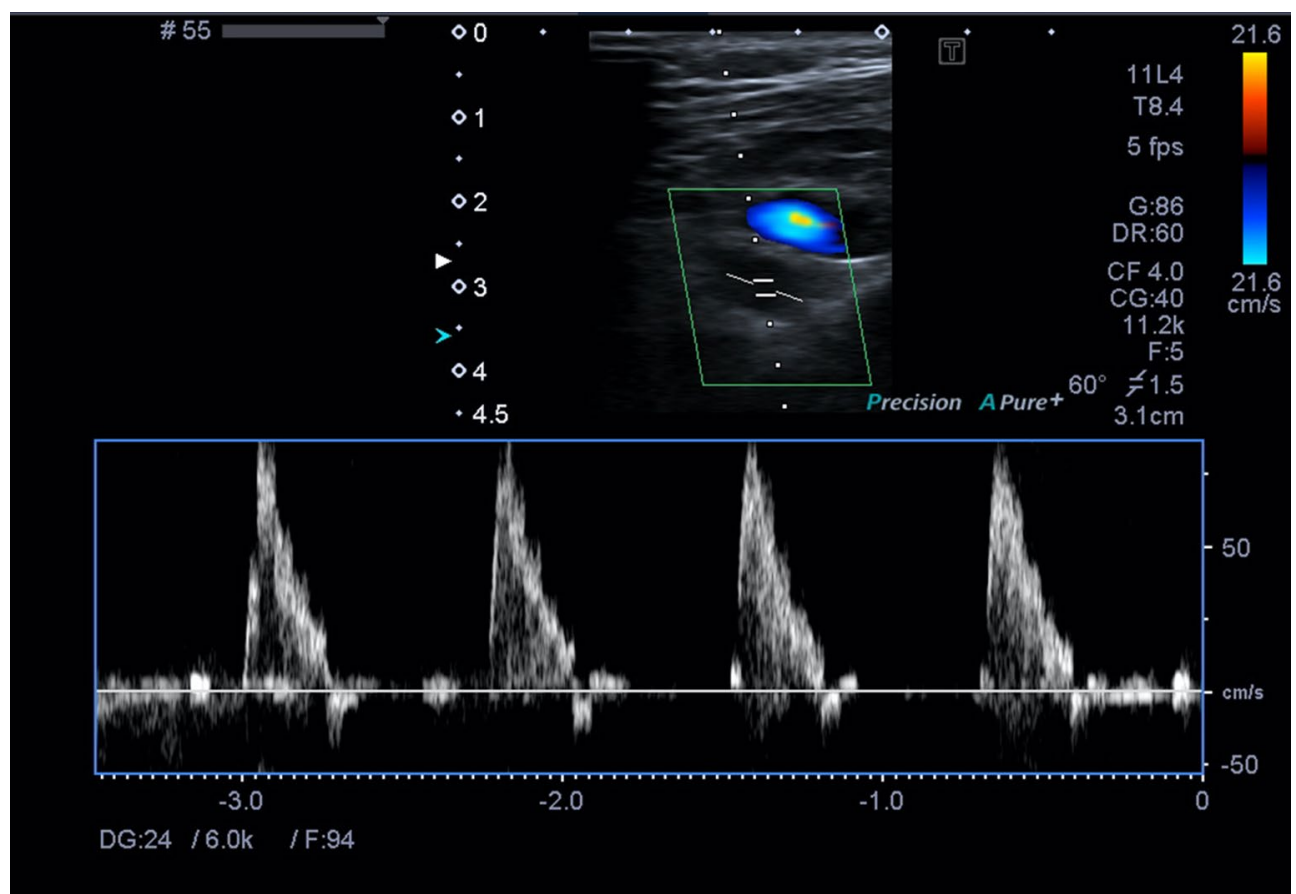

Figure 2. Normal color Doppler ultrasound image showing the subclavian artery findings at two months after the treatment.

\section{DISCUSSION}

Pectoralis minor syndrome is defined as compression of the neurovascular bundle at the retropectoralis minor space. Pain, numbness, tingling, and weakness are the common symptoms. Neural structures are more frequently affected than vascular structures in PMS. ${ }^{[3,4]}$ Vascular PMS, an infrequent condition, is characterized by axillary vein/artery obstruction due to compression caused by the PM muscle below the clavicle. Cyanosis and swelling of the involved upper extremity and discolored, cold and painful hand along with possible ischemia or possible numbness of fingers are seen. A feeling of fullness and tightness of the extremity usually occur. In non-thrombotic obstruction, the symptoms are intermittent, often appearing with elevation of the extremity and disappearing when the extremity is at rest. Symptoms are usually more intense and persistent, when thrombosis occurs. ${ }^{[5]}$

Pectoralis minor syndrome is rarely on a physician's differential diagnosis and, therefore, it is mostly neglected. Patients should be questioned about their occupation, exercise habits and sportive activities, looking for a cause of repetitive stress injury and history of a trauma. As the PM muscle attaches to the coracoid process of the scapula, repetitive shoulder and arm movements are the usual etiology of PMS as seen in this patient. ${ }^{[3]}$ Our patient was interested in rowing and playing tennis and her symptoms worsened after she exercised. She was initially suspected to have cervical radiculopathy. However, EMG evaluation and MRI of cervical spine were unremarkable.

A detailed history and physical examination are critical to determine the location of the compression. The PM muscle blocks are performed to confirm the diagnosis suspected based on clinical findings. The US-guided block is reliable, practical, and inexpensive and it allows visualizing neurovascular structures and avoiding complications. A short-acting lidocaine is often used in diagnostic blocks and $4 \mathrm{~mL}$ of $1 \%$ lidocaine is injected into PM muscle with a 45 -degree angle injection. A successful block is defined by the loss of the tenderness over the PM muscle within 1 to 2 min of injection, pain and paresthesia symptoms are expected to be reduced additionally. ${ }^{[6]}$ Odderson et al. ${ }^{[7]}$ suggested that anesthetic muscle blocks, with the help of Doppler USG, could be useful in localizing the neurovascular compression in TOS. Dynamic neuromusculoskeletal USG was also suggested to assist in the diagnosis of TOS/brachial plexopathy. Similarly, in our case, color Doppler USG with Adson maneuver revealed a decrease in the flow velocity in the axillary artery and an increase in the 
acceleration time. Since there was no thrombosis and acute arterial symptoms, we preferred conservative treatment. After the PM muscle block, PM stretching, shoulder stabilization, postural correction exercises and shoulder proprioceptive neuromuscular facilitation muscle stretching were initiated with activity modification. A significant improvement was found in the VAS 1 and QuickDASH 4.5 scores and her upper extremity arterial venous system color Doppler USG was normal after two months.

The treatment approach can be divided into operative and non-operative methods. Non-operative procedures are physical therapy, analgesics, muscle relaxants and anti-inflammatory drugs. ${ }^{[5]}$ When such conservative measures fail, PM tenotomy becomes the treatment of choice. ${ }^{[3]}$ Success of physical therapy depends on the onset of symptoms. Physical therapy that begins many months after the onset of symptoms has a lower percentage of success. ${ }^{[2]}$ Early diagnosis and treatment are important, particularly in vascular PMS.

In conclusion, one of the primary issues in TOS patients is to identify where the compression is and finding the exact cause of symptoms. The awareness and suspicion of PMS should be high in upper extremity problems to make the accurate diagnosis and early treatment.

\section{Declaration of conflicting interests}

The authors declared no conflicts of interest with respect to the authorship and/or publication of this article.

Funding

The authors received no financial support for the research and/or authorship of this article.

\section{REFERENCES}

1. Kuhn JE, Lebus V GF, Bible JE. Thoracic outlet syndrome. J Am Acad Orthop Surg 2015;23:222-32.

2. Sanders RJ, Hammond SL, Rao NM. Diagnosis of thoracic outlet syndrome. J Vasc Surg 2007;46:601-4.

3. Sanders RJ, Annest SJ. Pectoralis minor syndrome: Subclavicular brachial plexus compression. Diagnostics (Basel) 2017;7:46.

4. Aktas I, Kaya E, Akpinar P, Atici A, Unlu Ozkan F, Palamar $\mathrm{D}$, et al. Spasticity-induced Pectoralis minor syndrome: A case-report. Top Stroke Rehabil 2020;27:316-9.

5. Sanders RJ, Annest SJ. Thoracic outlet and pectoralis minor syndromes. Semin Vasc Surg 2014;27:86-117.

6. Bottros MM, AuBuchon JD, McLaughlin LN, Altchek DW, Illig KA, Thompson RW. Exercise-enhanced, ultrasoundguided anterior scalene muscle/pectoralis minor muscle blocks can facilitate the diagnosis of neurogenic thoracic outlet syndrome in the high-performance overhead athlete. Am J Sports Med 2017;45:189-94.

7. Odderson IR, Chun ES, Kolokythas O, Zierler RE. Use of sonography in thoracic outlet syndrome due to a dystonic pectoralis minor. J Ultrasound Med 2009;28:1235-8. 